\title{
A Novel Platform to Study Magnetized High-Velocity Collisionless Shocks
}

\author{
D. P. Higginson ${ }^{\mathrm{a}, *}$, Ph. Korneev ${ }^{\mathrm{b}, \mathrm{c}}$, J. Béard ${ }^{\mathrm{d}}$, S. N. Chen ${ }^{\mathrm{a}}$, E. d'Humières ${ }^{\mathrm{b}}$, H. Pépine ${ }^{\mathrm{e}}$, S. Pikuz ${ }^{\mathrm{f}}$, B. Pollock ${ }^{\mathrm{g}}$, \\ R. Riquier ${ }^{\mathrm{a}, \mathrm{h}}$, V. Tikhonchuk ${ }^{\mathrm{b}}$, J. Fuchs ${ }^{\mathrm{a}}$ \\ ${ }^{a}$ Laboratoire pour l'Utilisation des Lasers Intenses, UMR 7605 CNRS-CEA-École Polytechnique-Université Paris VI, 91128 Palaiseau, \\ France \\ ${ }^{b}$ University of Bordeaux-CNRS-CEA, CELIA, UMR5107, 33405 Talence, France \\ ${ }^{c} N R N U$ 'MEPhI', 115409 Moscow, Russian Federation \\ ${ }^{d} L N C M I, U P R$ 3228, CNRS-UJF-UPS-INSA, 31400 Toulouse, France \\ e INRS-ÉMT, 1650 bd. L. Boulet, J3X1S2 Varennes, Québec, Canada \\ ${ }^{f}$ Joint Institute for High Temperatures, RAS, 125412, Moscow, Russia \\ ${ }^{g}$ Lawrence Livermore National Laboratory, Livermore, California 94440, USA \\ ${ }^{h}$ CEA, DAM, DIF, 91297 Arpajon, France
}

\begin{abstract}
An experimental platform to study the interaction of two colliding high-velocity $(0.01$ to $0.2 \mathrm{c} ; 0.05$ to $20 \mathrm{MeV})$ proton plasmas in a high strength $(20 \mathrm{~T})$ magnetic field is introduced. This platform aims to study the collision of magnetized plasmas accelerated via the Target-Normal-Sheath-Acceleration mechanism and initially separated by distances of a few hundred microns. The plasmas are accelerated from solid targets positioned inside a few cubic millimeter cavity located within a Helmholtz coil that provides up to $20 \mathrm{~T}$ magnetic fields. Various parameters of the plasmas at their interaction location are estimated. These show an interaction that is highly non-collisional, and that becomes more and more dominated by the magnetic fields as time progresses (from 5 to $60 \mathrm{ps}$ ). Particle-in-cell simulations are used to reproduce the initial acceleration of the plasma both via simulations including the laser interaction and via simulations that start with preheated electrons (to save dramatically on computational expense). The benchmarking of such simulations with the experiment and with each other will be used to understand the physical interaction when a magnetic field is applied. Finally, the experimental density profile of the interacting plasmas is shown in the case without an applied magnetic magnetic field, so to show that without an applied field that the development of high-velocity shocks, as a result of particle-to-particle collisions, is not achievable in the configuration considered.
\end{abstract}

\section{Introduction}

A major motivation for the study of collisionless shocks is to understand how these shocks may accelerate ions to very high velocities via reflections of ions across the high electric and magnetic fields that can develop in the shocks. Such processes could be at the source of emissions observed from Supernova Remnants (SNRs) shocks. However, the possibility for such mechanism to be efficient remains the subject of intense debate and active research $[1,2,3,4,5]$. Hence, to have the possibility for the first time to study all these effects in the laboratory is a great opportunity to bring significant new results to compare with existing observations and to simulations of these phenomena.

The study of such phenomena has been the focus of several groups and the first interesting results have been obtained, mostly by using the collision of relatively slow $\left(\sim 10^{8} \mathrm{~cm} / \mathrm{s}\right)$ plasma streams produced by high-power, long-pulse lasers $[6,7,8,9,10,11]$. However, up to this

\footnotetext{
* Corresponding author

Email address: drew.higginson@polytechnique.edu (D. P. Higginson)
}

point the study of collisions with higher velocity flows has not, to our knowledge, been investigated. Such velocities are more relevant to astrophysical application but were not previously exploited because the characteristic crossing time of such laser-produced flows is too fast for the shock to develop. However, by strongly magnetizing the flows, we can, in principle, increase the shock formation rate and can produce and study these collisionless shocks associated with fast flows in the laboratory[12]. Additionally, magnetized flows are themselves of interest since astrophysical flows and shocks often are produced in a magnetized environments.

In this paper, we will detail an experimental setup that will be used to study these high-velocity, collisionless shocks. We will detail the energy spectra and number densities of ions accelerated via the Target Normal Sheath Acceleration (TNSA) mechanism[13, 14, 15]. This will be shown experimentally and then through simulations. The collision of the two TNSA plasmas will be studied without any applied magnetic field and, as expected, will show no shock generation or significant density increases at the collision point. We will use the plasma parameters derived from the simulations and experiments to predict the con- 


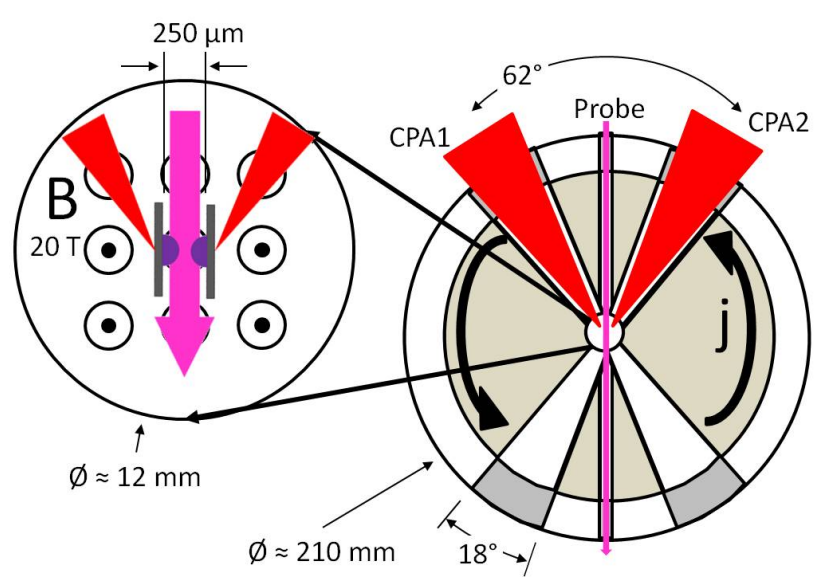

Figure 1: A top view of the experimental setup of the main, split and probe beams inside the Helmholtz coil. The full view (on the right) shows the various entrance bores within the coil to allow for multiple lasers to pass through the interaction region. The insert (on the left) shows a close-up of the target configuration in the center of the coil with the applied magnetic field perpendicular (upwards) to the interaction direction.

ditions of the collision during the application of a high (20 $\mathrm{T})$ external magnetic field.

\section{Experimental Setup using External Magnetic Field}

This experiment was performed at the Titan Laser Facility at the Lawrence Livermore National Laboratory using the short pulse laser beam in the split-beam configuration. This configuration splits the beam symmetrically so that the energy contained in each segment is relatively equal in energy $(\sim 60 \mathrm{~J}, 650 \mathrm{fs})$. The beams are then focused using two f/ 3 parabolas which resulted in two highintensity focal spots, which are oval in shape due to the semi-circular incoming beam: $\mathrm{FWHM}=5.8 / 13.6 \mu \mathrm{m}$ (vertical/horizontal), $I_{\max }=5.8 \times 10^{19} \mathrm{~W} / \mathrm{cm}^{2}$ (main beam) and FWHM $=4.4 / 11.6 \mu \mathrm{m}, I_{\max }=8.4 \times 10^{19} \mathrm{~W} / \mathrm{cm}^{2}$ (split beam). Both beams are timed, by using the overlapping of the beams to create interference fringes, to arrive at the same time on the targets shown in Figure 1.

The goal of this platform is to collide two high-velocity proton plasma flows in the presence of a strong, externally generated magnetic field. For this purpose, a highcurrent, Helmholtz coil was constructed at the Laboratoire National des Champs Magnétiques Intenses (LNCMI) in Toulouse. As shown in Figure 1, the wires are wrapped around the axis of the coil so that the magnetic field is created in a direction perpendicular to the velocity of the proton beams. The full diameter of the coil is $210 \mathrm{~mm}$.

The driver behind the Helmholtz coil is a $30 \mathrm{~kJ}, 20 \mathrm{kV}$, capacitor bank, which is able to provide enough current to provide a $20 \mathrm{~T}$ magnetic field at the center of the coil. The magnetic field is constant to within $2 \%$ of the peak value at distances up to $3 \mathrm{~mm}$ in radius from the center of the coil. In time, the magnetic field strength stays constant to

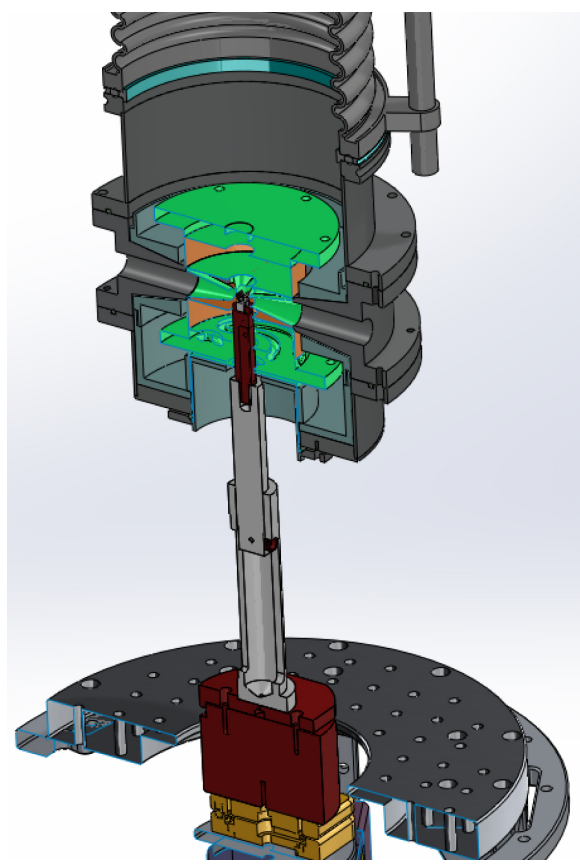

Figure 2: A graphical design of the coil with the target insertion system. The plastic target insertion system uses a telescoping arm to allow the targets to be inserted at the correct height, prior to precise alignment using the motorized stages.

within $2 \%$ for a duration of $50 \mu$ s. Thus the magnetic field can be considered as constant across both time and space in comparison to the distances and time scales of the laser interaction.

Figure 1 shows how the beam access into the coil is allowed by two conical bores separated by $62^{\circ}$. These bores have a total opening angle of $19^{\circ}$ and were fabricated so that the diameter of the cone would be $5 \mathrm{~mm}$ at the center of the coil. A constant-diameter access bore of $5 \mathrm{~mm}$ was centered between the two conical bores for use with a probe beam. Finally, an additional access from the bottom of the coil was used to allow the targets to be positioned within the coil. The targets were inserted by a plastic, telescoping target insertion support as shown in Figure 2, which allowed two targets to be inserted into the small $10 \mathrm{~mm}$ central region. The top of this telescoping mount, as shown in Figure 3 holds the two targets with two plastic target holders, these both allow the thin metal targets to be held in place and they also block some of the self-emission from the laser irradiated side of the target in order to make optical diagnosis more feasible. These target holders slide along a rail that allows the two targets to be positioned at different distances from each other.

\section{Ion Acceleration}

\subsection{Measurement of Proton Spectrum using Radiochromic Film Stacks}

To accelerate the high-energy protons, the two shortpulse lasers were focused onto $\mathrm{Al}$ targets of $4.5 \mu \mathrm{m}$ thick- 


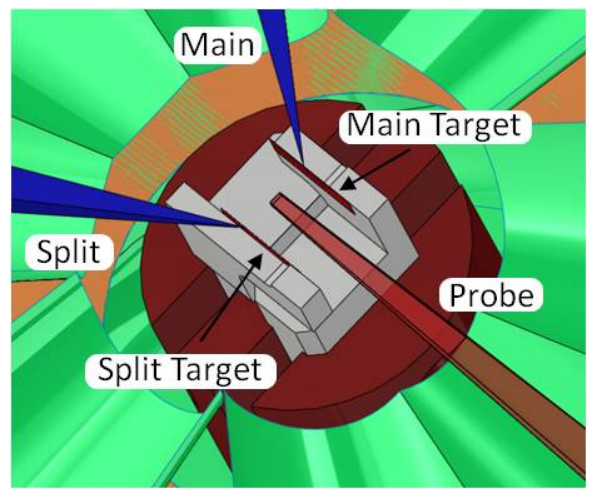

Figure 3: Graphical view of the top of the target insertion system. The two targets are aligned on a rail so that their positions can be adjusted to varying distance from each other.

ness. This accelerated protons via the target-normal-sheathacceleration (TNSA) mechanism, which has previously been studied in-depth[13, 14, 15, 16, 17].

The spectrum of the proton beam was measured (without external magnetic fields) using a stack of Radiochromic film (RCF) placed $35 \mathrm{~mm}$ from the solid target. The types of film used were HD (original version) and MD (original version), the films were cut to be 25 by $35 \mathrm{~mm}$. RCF is an absolutely calibrated film that increases in optical density when exposed to ionizing radiation. The RCF stack consisted of layers of HD ("v1") film followed by, the more sensitive, MD ("v1") film, with alternating layers of Polyethylene Terephthalate (PET). This method allowed the measurement of protons with energies above $1.8 \mathrm{MeV}$.

The proton energy spectrum is inferred from the RCF stack via the following steps (as also explained in Ref [18]):

1. Conversion of the experimentally measured optical density into energy deposition for each film.

2. Construct a response matrix giving energy deposition in each film as a function of (mono-energetic) proton kinetic energy. This is done by modeling the propagation of protons through the RCF stack using the continuous slowing down approximation (CSDA) using stopping powers from the code SRIM[19]. In this model, we neglect both lateral and longitudinal straggling, which is a good assumption considering the shallow depth of the film and the relatively straight trajectories of protons. We also consider all protons to be traveling normal to the RCF stack surface, which is valid due to the low angles of incidence.

3. The experimental data is then fit (via the response function) assuming an exponential-decay-like spectrum

4. Finally, a response function is constructed using this fitted spectrum. This allows the total number of protons passing though each film to be inferred as well as their average energy.

The proton energy spectrum is shown in Figure 4. The best exponentially fits, where $\mathcal{H}(x)$ is the Heaviside step-

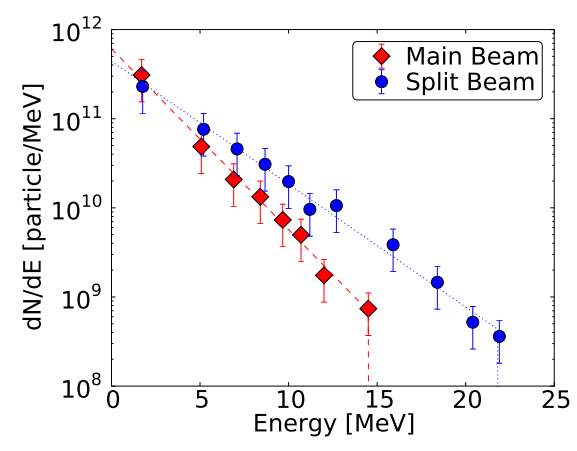

Figure 4: Experimentally measured energy spectrum of the proton beams from the main beam (diamonds, Shot9) and the split beam (circles, Shot11). These are fit with exponentially decaying functions with dashed and dotted lines for the main and split beams, respectively. Error bars come from the expected $\sim 50 \%$ shot-to-shot variability.

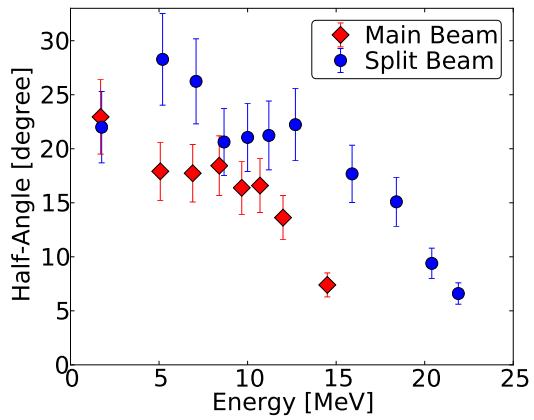

Figure 5: Experimentally measured divergence angles of the proton beams from the main (diamonds, Shot9) and split (circles, Shot11) beams. The error bars are derived from the non-uniformity of the beam shape.

function, are shown given by the equation below,

$$
\frac{d N}{d \mathcal{E}}(\mathcal{E})=N_{0} \exp (-\mathcal{E} / T) \times \mathcal{H}\left(\mathcal{E}_{\text {max }}-\mathcal{E}\right)
$$

the best fits to the data were found to be, for the main beam : $N_{0}=6.1 \times 10^{11}, T=2.1 \mathrm{MeV}, \mathcal{E}_{\max }=14.5 \mathrm{MeV}$, and for the split beam : $N_{0}=4.4 \times 10^{11}, T=3.1 \mathrm{MeV}$, $\mathcal{E}_{\text {max }}=21.9 \mathrm{MeV}$.

To understand the evolution of the proton density as it propagates over a given distance it is important to understand the angular divergence of the beam. To this end, the half-width-at-half-maximum (HWHM) of the proton beam was measured on each of the films. This was then converted into an angular divergence half-angle using the known $(35 \mathrm{~mm})$ distance of the film stack from the main [2\$arget and assuming a point source, which should be a good approximation as true source size should not exceed $100 \mu \mathrm{m}$. The half-angle of the proton beam is shown in Figure 5. One can see that there is a strong dependence of the divergence angle on the energy of the protons. However, at the lower energies (2 to $10 \mathrm{MeV}$ ) the divergence half-angle is more-or-less constant at around $20^{\circ}$, however this may diminish again at lower energies[21, and references therein]. 


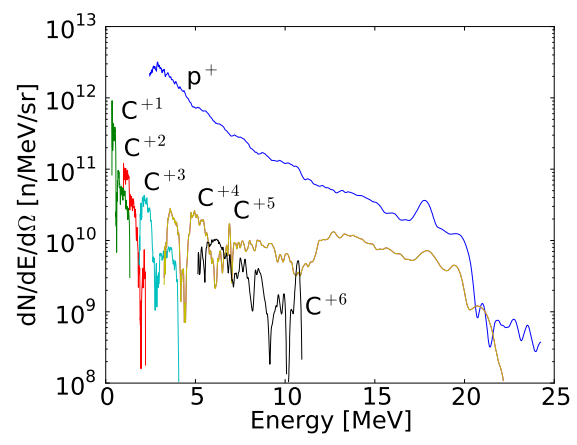

Figure 6: Proton and carbon ion spectra measured using the Thomson Parabola, from the split beam incident on a solid $4.5 \mu \mathrm{m} \mathrm{Al}$ foil (Shot55). The minimum cutoffs of the spectra are set by the limits of the TP and are not physical phenomena.

\subsection{Measurement of Ion Spectrum via Thomson Parabola}

In general, the TNSA mechanism preferentially accelerates protons over other ion species for two main reasons 1) the higher charge-to-mass ratio of protons and 2) the fact that the protons contribution comes from a hydrocarbon contaminant layer on the surface of the target, which makes them the first to accelerate, thus diminishing the field. However, in order to make sure that the interaction of the two plasmas in our experiment is dominated by the protons, the other ion species were also be measured. To this end, we used a Thomson Parabola to study the acceleration of multiple ion species.

A Thomson Parabola (TP)[18] uses parallel electric and magnetic fields to deflect ions, passing through a pinhole before the fields, dependent on their energy and chargeto-mass ratio. This allows different ion species to be separated and to determine the energy spectrum of each. The $100 \mu \mathrm{m}$ pinhole was placed at a distance of $200 \mathrm{~mm}$ from the main target. The magnet was $50.8 \mathrm{~mm}$ long with a field strength of $0.605 \mathrm{~T}$. The electrode of a similar length with an applied voltage of $6 \mathrm{kV}$. A Fujifilm TR imaging plate with a $90 \mathrm{~mm}$ length along the magnetic dispersion axis, was placed at $628 \mathrm{~mm}$ from the magnet and electrode to give an energy resolution down to $2.5 \mathrm{MeV}$ for protons.

The resultant spectra from the TP are shown in Figure 6 , which shows a similar spectrum of the protons as reproduced from the RCF stack. Note that the distribution of carbon ions is from 10 to 100 times lower than the number of protons. For this reason, we do not expect the carbon ions (or any other ions) to play a major role in dynamics of the colliding plasmas. Note that, due to the limited size of the imaging plate, there is a minimum energy that can be resolved by the TP for a given geometry, as a function of the charge-to-mass ratio. This is the reason that some of the ion spectra is not continuous down to lower energies.

\section{Estimation of Ion Density}

Now that the proton energy spectrum has been deduced, this information is used to get an estimation of the expected values of density for the interaction. For this we do a few simple mathematical transformations. First, the energy spectrum is transformed into a velocity spectrum, where $v$ is the proton velocity, $\mathcal{E}$ is the proton kinetic energy and $m_{\mathrm{p}}$ is the proton mass. This assumes that the protons are accelerated instantaneously at the start of the laser pulse, which is not true but should only add an error on the order of the laser pulse length (0.65 ps). The calculation is done non-relativistically, as the maximum proton energy is less than $25 \mathrm{MeV}$.

$$
v=\sqrt{\frac{2 \mathcal{E}}{m_{\mathrm{p}}}} ; \quad \frac{d N}{d v}=\frac{d N}{d \mathcal{E}} \times \sqrt{2 \mathcal{E} m_{\mathrm{p}}}
$$

This velocity spectrum is then transformed into a total number profile based on the time, $t$, of interest, as a function of the distance, $r$, from the target.

$$
\begin{aligned}
r & =v t=\sqrt{\frac{2 \mathcal{E}}{m_{\mathrm{p}}}} \times t \\
\frac{d N}{d r} & =\frac{d N}{d v} \times \frac{1}{t}=\frac{d N}{d \mathcal{E}} \times \frac{\sqrt{2 \mathcal{E} m_{\mathrm{p}}}}{t}
\end{aligned}
$$

To calculate the density of the ions it is necessary to know the spatial extent of the plasma. For this the halfangle, $\theta_{1 / 2}$, of the beam, as deduced from the RCF stack is used. In this derivation, we used both the raw data (i.e. points in Figure 4) and the fit data (i.e. lines in Figure 4). For the raw data, the half-angle used was the measured value (i.e. points in Figure 5), for the fit data a constant half-angle of $20^{\circ}$ is used. An initial diameter, $d_{0}$, of $10 \mu \mathrm{m}$ is used (the laser spot size) to give the diameter as a function of the distance from the target, $r$. Recall that here the diameter $d$ is the full-width-at-half-maximum (FWHM) of the beam.

$$
d_{\mathrm{FWHM}}=d_{0}+2 r \tan \theta_{1 / 2}
$$

If we consider the beam to be a $2 \mathrm{D}$ gaussian, which is a good approximation for the proton beams, $I(R)=$ $I_{0} \exp \left(\frac{-R^{2}}{2 \sigma^{2}}\right)$, where $I$ is the intensity of the signal, the $R$ is the radial direction, and $\sigma$ is the characteristic width $\left(d_{\mathrm{FWHM}}=\sigma \sqrt{8 \ln 2}\right)$. Then the integrated signal, $T$, is related to the peak, $I_{0}$, by $T=2 \pi I_{0} \sigma^{2}=\frac{2 \pi}{8 \ln 2} I_{0} d_{\mathrm{FWHM}}^{2}$. Thus we can relate the peak density to the total number by the following relation.

$$
\frac{d N}{d V}=\frac{d N}{d r} \times \frac{8 \ln 2}{2 \pi d_{\mathrm{FWHM}}^{2}}
$$

Thus using these considerations we can estimate the density profile as the plasma expands. In Figure 7, the peak density profiles at three different times (5, 15, and $60 \mathrm{ps}$ ) are shown. These represent different times of interest for the plasma interaction. It is important to state that raw data points are only visible in the first plot at 5 ps. Therefore the subsequent density profiles are based 
on the exponential fits to the data and will be entirely dependent on the extrapolation of these fits across a region of energy that was not measured directly experimentally. The uncertainty in these profiles is found to be $52 \%$ by the addition in quadrature of the uncertainties of the proton number and beam divergence. To show this uncertainty, two gray lines are drawn to show the variation in the shape of the profile by adding this $52 \%$ uncertainty to the main beam profile and subtracting the $52 \%$ uncertainty from the split beam profile, or the inverse. Thus this gives an idea of the ways that the shape of the density profiles could be modified.

We note that the peaks in the density profile (Fig. 7) are due to the transportation of the exponential energy distribution into a velocity distribution. This transformation adds a factor of $\sqrt{\mathcal{E}}$, which drives the velocity (and thus the energy) distribution to zero at the beginning.

We see that the density changes significantly over the range of interest from a few $10^{18} \mathrm{n} / \mathrm{cm}^{3}$ at early times to a few $10^{16} \mathrm{n} / \mathrm{cm}^{3}$, a factor of around 100. At the same time, the velocity of the interacting particles is changing, thus giving an interacting plasma that evolves quickly in time. In the next section, we will go into detail of how the plasma parameters change with time.

\section{Expected Plasma Parameters}

\subsection{General Case}

In order to understand the physical regime of our experiment, we have calculated several of the physical parameters that we expect during the time scale of our experiment, as shown in Table 1 . The velocities are derived from the times that ions (protons) would take to move to the center of the interaction region (i.e. $125 \mu \mathrm{m}$ ) if they start at time zero with the given velocity. Of course, the protons will not be accelerated instantaneously, however this acceleration time should be on the order of the laser pulse duration[16](i.e. 1.3 times the duration). Thus this will add some uncertainty to the estimation, which is at maximum $18 \%$ in the short ( $5 \mathrm{ps}$ ) time scale, which would make the real proton velocity higher than that indicated on the table. For the density, the peak values estimated from the previous section are used. The electron temperature is taken using the analysis preformed in Ref [22] for the adiabatic expansion of a thin foil, with an initial laser-accelerated hot-electron density of $10^{19} \mathrm{n} / \mathrm{cm}^{3}$ $\left(\omega_{\mathrm{pi}}=0.13 \mathrm{ps}\right)[23]$ and an initial electron temperature of $2.5 \mathrm{MeV}$, which comes from the average proton slope temperature. We use the $1 \mathrm{MeV}$ temperature curve presented in FIG 2 of Ref [22] to determine the temporal evolution of the electron temperature. For values that are beyond this curve, we use a fit to a power-law curve, as suggested in the paper.

Note that the plasmas are highly non-collisional. The collisional mean-free-path (mfp) is much more than a few meters, while the maximum length scale in the experiment
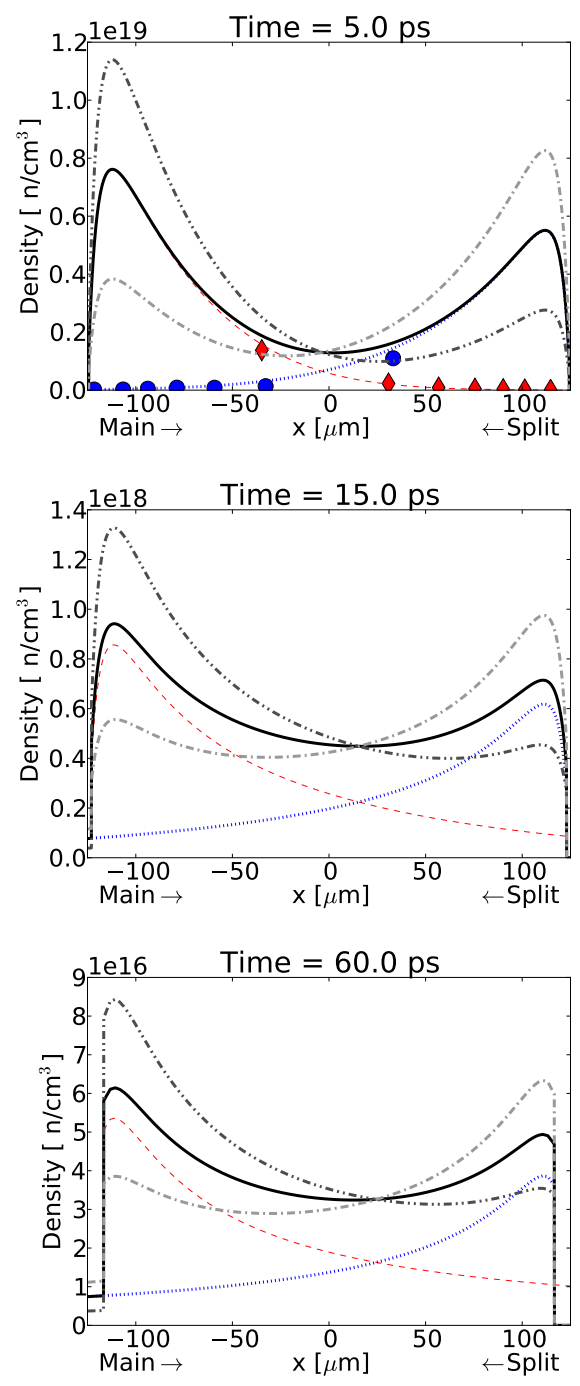

Figure 7: Estimates of the ion density at the indicated times of the colliding proton plasmas. The main beam comes from the left. Diamonds (main) and circles (split) show the raw data (only seen in the first plot). The dashed (main), dotted (split) and solid (summation of both) lines show the exponential fits. The gray dash-dot and dash-dot-dot lines show potential summation profiles when the the split profile is increased and the main profile is decreased, or the inverse, respectively, by the $52 \%$ uncertainty. 
in $250 \mu \mathrm{m}$. Also, since the plasma is quite non-resistive, we expect the magnetic fields to be 'frozen-in' to the plasma and thus to be advected along with the plasma flow. As shown previously in simulations [24, 25], this can lead to the accumulation of magnetic fields that increase up to $20 \mathrm{x}$ higher than the original field strength due to the compression of the field.

\begin{tabular}{c|cccc} 
& $\begin{array}{c}\text { Early } \\
\text { Time }\end{array}$ & $\begin{array}{c}\text { Mid } \\
\text { Time }\end{array}$ & $\begin{array}{c}\text { Late } \\
\text { Time }\end{array}$ & \\
\hline Time & 5 & 15 & 60 & $\mathrm{ps}$ \\
Length & 125 & 125 & 125 & $\mu \mathrm{m}$ \\
$v_{\text {ion }}$ & 25 & 8.3 & 2.1 & $\mu \mathrm{m} / \mathrm{ps}$ \\
& 0.08 & 0.03 & 0.007 & $\mathrm{v} / \mathrm{c}$ \\
$\mathcal{E}_{\text {k,ion }}$ & 3300 & 360 & 23 & $\mathrm{keV}$ \\
$T_{\mathrm{e}}$ & 170 & 20 & 1.4 & $\mathrm{keV}$ \\
Density & $2 \times 10^{18}$ & $5 \times 10^{17}$ & $4 \times 10^{16}$ & $\mathrm{n} / \mathrm{cm}^{3}$ \\
Coll. mfp & $1 \times 10^{7}$ & $5 \times 10^{5}$ & $3 \times 10^{4}$ & $\mathrm{~cm}$
\end{tabular}

Table 1: Estimates of parameters of the TNSA plasmas at different times during the interaction. The values are, in order, the time of interaction, distance of the interaction, the velocity of the proton that should be at the center of the interaction, the proton velocity, the proton ion kinetic energy, electron temperature, the peak density at the center of the interaction, and the collisional mean-free-path. We expect the uncertainty in these values to be on the order of $50 \%$ due to the shot-to-shot variation in proton number.

\subsection{Magnetized Case}

As the purpose of this experimental configuration is to collide plasmas in an external magnetic field, we will now expand upon the previous estimates to create an understanding for the plasma parameters that should be present in a magnetized case.

The pulsed Helmholtz coil is capable of delivering a magnetic field of 20 T. However, previous work[24] has shown that such a field can be compressed considerably, since the field are transported along with the plasma flows themselves. In these simulations, the maximum fields achieved by this compression were on the order of $500 \mathrm{~T}$. Thus to understand the value that may be present in the real experiment, we will look at magnetic field values of both 20 and $500 \mathrm{~T}$.

The results of our analysis are shown in Table 2 for the three different timescales considered previously $(5,15$, $60 \mathrm{ps}$ ). One can see considerable difference in how the plasmas react to the magnetization as time progresses. At early time, the thermal pressure of the plasma is obviously dominant over the pressure exerted by the magnetic fields. While there may be some effect modification of the electron trajectory by the magnetic field, this should be highly dependent on the value of the field and the amount of compression achieved.

At the middle time, the thermal pressure has decreased and thus the magnetic pressure may begin to play a role, however the importance of this role is governed by the amount of compression achieved and may be minimal.
However, the kinetic effects of the field on the electrons should begin to become important, as even at minimal (pre-compressed) values of the magnetic field, the Larmor radius begins to have a small size relative to the interacting system.

Finally, at the late time, the plasma begins to become magnetized. Even at lower magnetic field values, the magnetic pressure is of a similar order of the thermal pressure, thus with even minimal amounts of compression, this magnetic pressure may become dominate. Additionally, the electron Larmor radii should be quite small compared to the size of the system. Interestingly, at this point, depending on the amount of magnetic field compression, the ions may also experience some deviation due to large values of the magnetic field.

\begin{tabular}{|c|c|c|c|}
\hline Early Time & $\mathrm{B}=20 \mathrm{~T}$ & $\mathrm{~B}=500 \mathrm{~T}$ & \\
\hline P, Thermal & $4 \times 10^{10}$ & $4 \times 10^{10}$ & $\mathrm{~Pa}$ \\
\hline P, Magnetic & $2 \times 10^{8}$ & $1 \times 10^{11}$ & $\mathrm{~Pa}$ \\
\hline Beta & 260 & 0.4 & \\
\hline Ion $r_{L}$ & 13000 & 520 & $\mu \mathrm{m}$ \\
\hline Electron $r_{L}$ & 75 & 3 & $\mu \mathrm{m}$ \\
\hline \multicolumn{4}{|l|}{ Mid Time } \\
\hline P, Thermal & $1 \times 10^{9}$ & $1 \times 10^{9}$ & $\mathrm{~Pa}$ \\
\hline P, Magnetic & $2 \times 10^{8}$ & $1 \times 10^{11}$ & $\mathrm{~Pa}$ \\
\hline Beta & 9.2 & $1.5 \times 10^{-2}$ & \\
\hline Ion $r_{L}$ & 4300 & 180 & $\mu \mathrm{m}$ \\
\hline Electron $r_{L}$ & 24 & 1.0 & $\mu \mathrm{m}$ \\
\hline \multicolumn{4}{|l|}{ Late Time } \\
\hline P, Thermal & $8 \times 10^{6}$ & $8 \times 10^{6}$ & $\mathrm{~Pa}$ \\
\hline P, Magnetic & $2 \times 10^{8}$ & $1 \times 10^{11}$ & $\mathrm{~Pa}$ \\
\hline Beta & $4.9 \times 10^{-2}$ & $7.9 \times 10^{-5}$ & \\
\hline Ion $r_{L}$ & 1100 & 44 & $\mu \mathrm{m}$ \\
\hline Electron $r_{L}$ & 6.3 & 0.25 & $\mu \mathrm{m}$ \\
\hline
\end{tabular}

Table 2: Estimates of parameters related to the magnetization of the TNSA plasmas at different times (5, 15, $60 \mathrm{ps)}$ during the interacdion. Values shown, in order, are the thermal pressure, the magnetic pressure, the magnetic beta parameter (thermal/magnetic pressure), the proton ion Larmor radius, and the electron Larmor radius.

Thus as the plasma evolves in time the importance of the magnetization of the plasma increases. By looking at these difference time scales, we thus get an idea of how such different parameters will affect that evolution of the two-plasma interaction.

\section{Simulation and Reproduction of Initial (Non- magnetized) Ion Spectrum}

To make a connection between theory and experiment, we used Particle-in-Cell 2D3V simulations of the laser interaction with a composite target. These simulations used the code PICLS [26]. The target is $0.5 \mu \mathrm{m}$ thick and composed of Carbon ions, protons and electrons. The target density is 300 times the critical density. The plasma is 


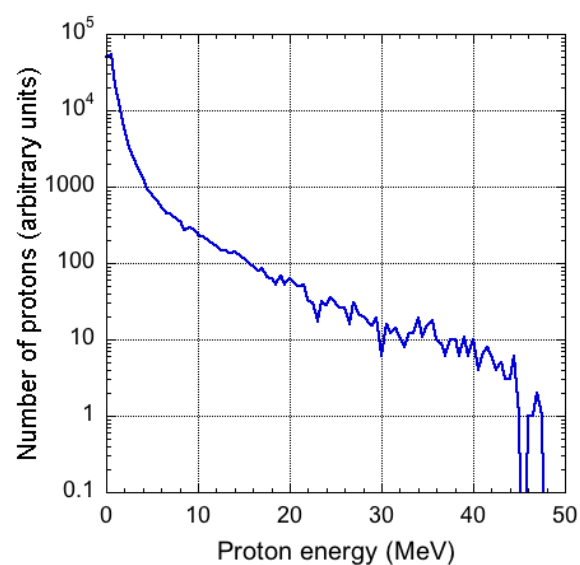

Figure 8: Proton energy spectrum obtained modeling the laserplasma interaction directly in the 2D PICLS simulation after 1.9 ps.

located $70 \mu \mathrm{m}$ from the left side in the $176 \times 160 \mu \mathrm{m}$ simulations box. The spatial and time steps are respectively $\Delta x=\Delta y=15 \mathrm{~nm}$ and $\Delta t=0.05 \mathrm{fs}$

The laser pulse was defined to be the same as in the experiment. Ionization process was not considered in simulation, the initial state of the target was plasma-like medium with cold ions and electrons, distributed in a way to neutralize ion charges. On the front side of the target we set nanoplasma, composed from $\mathrm{CH} 2$ plasma with an exponentially decrease and a characteristic width of $1 \mu \mathrm{m}$.

In figure 8 we present the results for the ion spectra. The maximum energy obtained in this simulation is higher than the one measured in the experiments which is to be expected from a $2 \mathrm{D}$ simulation confining hot electrons to the simulation plane. This spectrum can be fit with a double exponential spectrum with two slope-temperatures of $0.7 \mathrm{MeV}$ (mostly for energies below $10 \mathrm{MeV}$ ) and $7.5 \mathrm{MeV}$ (above $10 \mathrm{MeV}$ ), compared to the $2.5 \mathrm{MeV}$ temperature found experimentally.

\section{Simulations with Heated Electrons}

For the further high-resolved numerical studies we need to distinguish, which is the most important part from TNSA plasma, obtained in experiment. Subsequent studies will focus on the interaction of the two plasma flows (with or without applied magnetic fields) and so the ability to run simulations at long timescales is very important. It is evident, that taking into account all the target components will obstruct the physical understanding of the initial stage of flows interaction. In this section we compare the proton spectrum, obtained in experiment and in the previous full-target simulation, with the simplified model, described below. We expect this model to reproduce the proton spectrum, which much less computational expense than the laser-simulations allowing us to solve upcoming, long timescale, numerical problems.

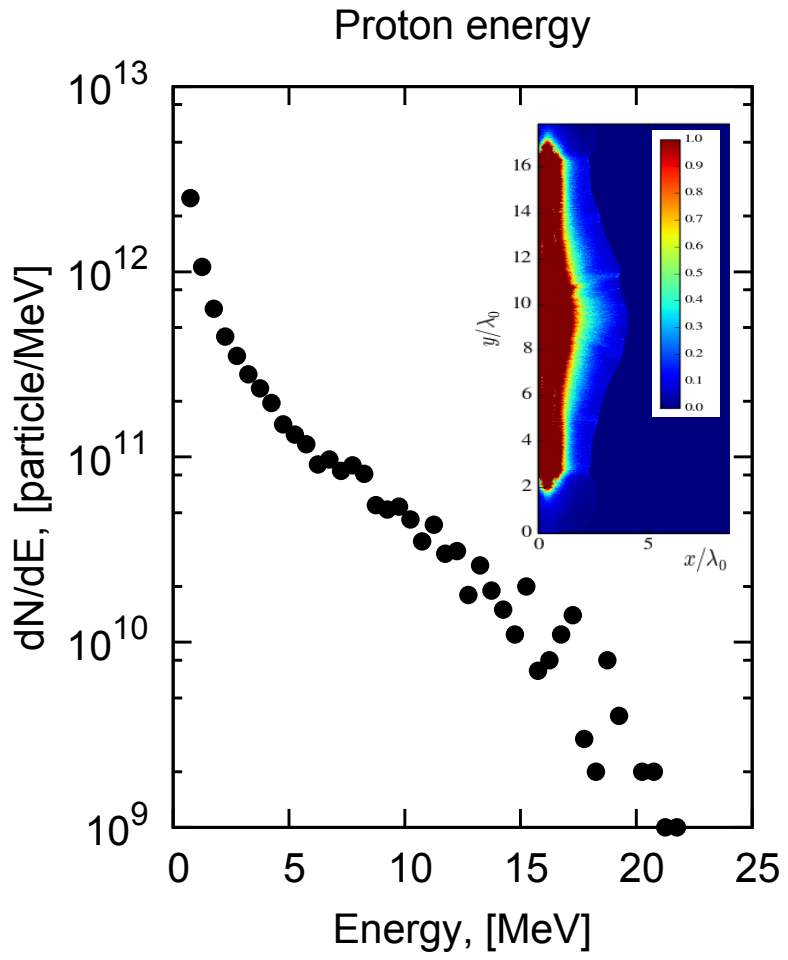

Figure 9: Proton energy spectrum from PIC simulations for an expansion of plasma slab with cold protons and heated electrons. Electron temperature (in the center) $T_{e}=1.87 \mathrm{Mev}$, slab width $2 \mu \mathrm{m}$. The inset show a picture of the electron density in the simulation at the time of $2.5 \mathrm{ps}$ timesteps. The colorbar is in units of $10^{19} \mathrm{n} / \mathrm{cm}^{3}$. The spatial scale is normalized to the spatial step in the simulation of $10.6 \mu \mathrm{m}$. 
The most important numerical problem, as we see it, is a wide range of plasma density in case of two flows collision. As we know from experimental measurements, in the interpenetration region, plasma has a density of about $\sim 10^{16-18} \mathrm{~cm}^{-3}$. Less dense parts may be also important in the case of an applied ambient magnetic field before the interpenetration process starts, because it accumulates the ambient magnetic field during the propagation and brings it to the interaction region $[25,24]$. At the same time, the low energy part of the expanding plasma, which comes to the interaction region later, has the initial density, comparable to the solid body, $\sim 10^{21-23} \mathrm{~cm}^{-3}$. This high density demands high spacial resolution, and at the same time, the very high number of particles to have a good statistics in the low density interaction region.

Our simplified plasma source for study a plasma flows collision is based on electron-proton plasma, contained in a thin (few microns) plate, with a temperature profile set for electrons, while ions are considered cold in the initial time moment. The plate density is $n \approx 3 \times 10^{21} \mathrm{~cm}^{-3}$ with a plate width of $2 \mu \mathrm{m}$. The results of the free expansion of such a target is shown in figure 9. It qualitatively reproduces the experimental spectrum for protons, demonstrates density decrease in 3 orders for energy range from 1 to $20 \mathrm{MeV}$.

\section{Measured Density Profile in non-Magnetized Case}

To analyze the density profiles of the colliding plasmas interferometry measurements were taken using a shortpulse $(650 \mathrm{fs})$ probe beam at $1 \omega(1054 \mathrm{~nm})$ in a MachZehnder geometry. Much care was taken to keep the optics free of debris to preserve an optimal phase front, as, due to the low densities present in the experiment, the expected phase shifts are on the order of $1-10 \%$ of the fringe spacing. The fringe spacing was $34 \mu \mathrm{m}$. The interference fringes were analyzed using the wavelet analysis tool within the image analysis toolbox of the code Neutrino[27].

Figure 10 shows an image taken by the inferometer of the TNSA plasma generated by the two short pulse lasers. The image is taken of the rear sides of the targets $(4.5 \mu \mathrm{m}$ $\mathrm{Al}$ ) separated by $250 \mu \mathrm{m}$ so that the laser pulses are not visible. The beams are incident across the horizontal direction (called 'propagation direction' in the figure) with the main beam coming from the left side and the split beam coming from the right side. The image shows an in-homogeneity, in that the right side of the image has a much higher observed fringe shift. Also, the left side of the image does not seem to very symmetric in the lateral dimension. The reason for this lateral difference is due mostly to the quality of the fringe shifts observable, because this data was taken near the edges of the foil it is subject to some diffraction and to being blocked by the edge of the foil holder, which will tend to blur out the shift of the fringes locally.

Figure 11 shows a lineout taken across the horizontal plane of this image centered at the center of the beams.

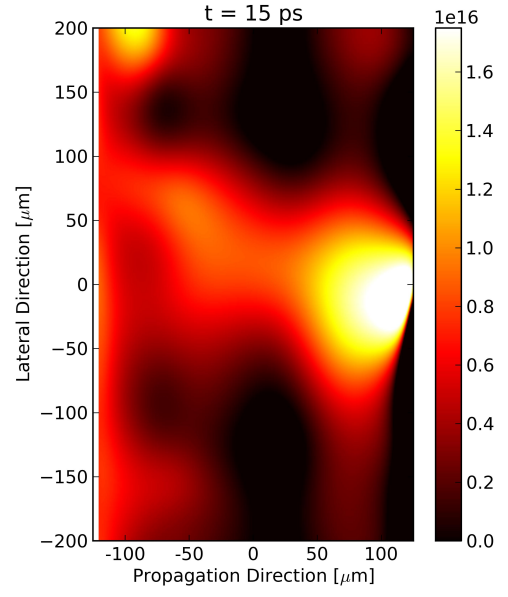

Figure 10: Integrated density image of two colliding rear-side TNSA plasmas coming from the left (main beam) and the right (split beam) without an external magnetic field (Shot58). Taken using the MachZehnder interferometer at 15 ps from the short-pulse interaction. The color axis is shown in units of integrated density of $\mathrm{n} / \mathrm{cm}^{2}$, which corresponds to fringe shifts of $10^{16} \mathrm{n} / \mathrm{cm}^{2} \rightarrow \Delta L / L=4.7 \%$.

Notice that the density from the split beam target is about a factor of $2 \mathrm{x}$ higher than the split beam. This difference may be due to on-shot differences between the two beams or simply shot-to-shot variation of the ion acceleration.

Notice that in there is no significant observed density increase at the center of the interaction region. This suggests that, without an external magnetic field, that the density of the two beams is not high-enough to form visible shock when they interact without the presence of an external magnetic field.

We also show in Figure 11, the comparison between the estimated integrated density profiles from the RCF spectrum as derived in Section 4. Notice that this line matches the experimentally measured quantities quite well at the center of the interaction. However, at the edges of the interaction the peak values deviate significantly. There are a few possible explainations for this, first of all we did not directly measure the proton energy or divergence spectra at these lower energies, which creates uncertainties in the fits. Additionally, at these distances very close to the target, the density from other ions accelerated in the interaction (e.g. Carbon) may be higher, due to the fact that these ions are more bunched together than the protons due to their lower velocity.

\section{Conclusions and Perspectives}

We have introduced an experimental platform to study the interaction of two colliding high-velocity ( 0.01 to 0.25 c) TNSA proton plasmas with or without an applied external magnetic field. The expected plasma parameters from such interactions have been estimated in the presence of an external magnetic field. Such estimations show 


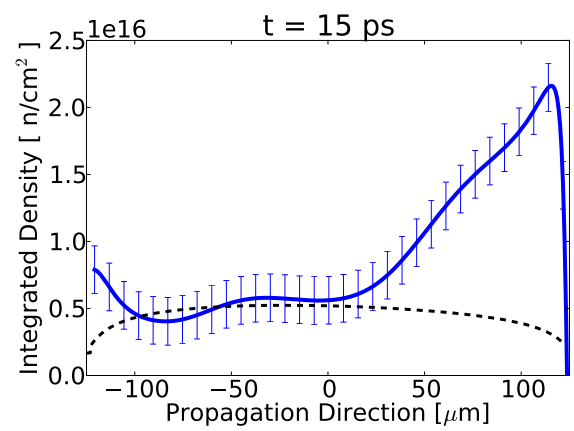

Figure 11: Horizontal lineout of the integrated density profile from the interferometer at $15 \mathrm{ps}$ from the short-pulse interaction in the solid line with error bars. The left and right sides correspond to the main and split beams, respectively. The dotted line is the expected integrated density inferred from the RCF spectrum as derived in Section 4.

a plasma that is highly collisionless, so that any interaction should be due to collective effects and not to collisions. We can also expect, according tho the theory presented in Ref [24], that the magnetic fields may be advected with the plasma flow, which should lead to the compression and enhancement of the field. Looking at the relative strength magnetic field, both via fluid and kinetic effects, shows a plasma that should be dominated by magnetic forces at given times in its evolution.

We have used Particle-in-cell simulations to reproduce the spectrum of the interacting protons, using both direct modeling of the laser-plasma interaction and using a simplified model using heated electrons, which should be much less computationally expensive. With these tools, we expect to be able to understand and identify the physics relevant to the two-plasma interaction.

We believe that this setup should be extremely useful to enhance our understanding of the role of collisionless shocks and how these shocks may be affected by external magnetic fields to either create or simulate a stronger interaction.

\section{Acknowledgments}

The authors thank S. Andrews, J. Bonlie, C. Bruns, R. C. Cauble, D. Cloyne, R. Costa and the entire staff of the Titan Laser and the Jupiter Laser facility for their support during the experimental preparation and execution. Thanks goes to Dr. T. Vinci for development of the Neutrino code[27] and advice on its use. This work was supported by ANR Blanc Grant $n^{\circ}$ 12-BS09-025-01 SILAMPA. This work was performed under the auspices of the U.S. Department of Energy by Lawrence Livermore National Laboratory under Contract DE-AC52-07NA27344. This work was granted access to the HPC resources of CINES under the allocation 2014-056129 made by GENCI (Grand Equipement National de Calcul Intensif).

[1] S. F. Martins, R. A. Fonseca, L. O. Silva, W. B. Mori, Ion dynamics and acceleration in relativistic shocks, The Astrophysi- cal Journal Letters 695 (2) (2009) L189.

URL http://stacks .iop.org/1538-4357/695/i=2/a=L189

[2] A. Bret, Weibel, two-stream, filamentation, oblique, bell, b2uneman... which one grows faster?, The Astrophysical Journal 699 (2) (2009) 990.

[3] L. O. Silva, R. A. Fonseca, J. W. Tonge, J. M. Dawson, W. B. Mori, M. V. Medvedev, Interpenetrating plasma shells: Nearequipartition magnetic field generation and nonthermal particle acceleration, The Astrophysical Journal Letters 596 (1) (2003) L121.

[4] L. Sironi, A. Spitkovsky, Particle acceleration in relativistic magnetized collisionless electron-ion shocks, The Astrophysical Journal 726 (2) (2011) 75. URL http://stacks. iop.org/0004-637X/726/i=2/a=75

[5] M. E. Dieckmann, A. Meli, P. K. Shukla, L. O. C. Drury, A. Mastichiadis, Two-dimensional pic simulations of ion beam instabilities in supernova-driven plasma flows, Plasma Physics and Controlled Fusion 50 (6) (2008) 065020.

URL http://stacks. iop.org/0741-3335/50/i=6/a=065020

[6] Y. Kuramitsu, Y. Sakawa, T. Morita, C. Gregory, J. Waugh, S. Dono, H. Aoki, H. Tanji, M. Koenig, N. Woolsey, et al., Time evolution of collisionless shock in counterstreaming laserproduced plasmas, Physical review letters 106 (17) (2011) 175002.

[7] N. Kugland, D. Ryutov, P. Chang, R. Drake, G. Fiksel, D. Froula, S. Glenzer, G. Gregori, M. Grosskopf, M. Koenig, et al., Self-organized electromagnetic field structures in laserproduced counter-streaming plasmas, Nature Physics 8 (11) (2012) 809-812.

[8] H.-S. Park, D. Ryutov, J. Ross, N. Kugland, S. Glenzer, C. Plechaty, S. Pollaine, B. Remington, A. Spitkovsky, L. Gargate, et al., Studying astrophysical collisionless shocks with counterstreaming plasmas from high power lasers, High Energy Density Physics 8 (1) (2012) 38-45.

[9] J. Ross, S. Glenzer, P. Amendt, R. Berger, L. Divol, N. Kugland, O. Landen, C. Plechaty, B. Remington, D. Ryutov, et al., Characterizing counter-streaming interpenetrating plasmas relevant to astrophysical collisionless shocksa), Physics of Plasmas (1994-present) 19 (5) (2012) 056501.

[10] T. Morita, Y. Sakawa, Y. Kuramitsu, S. Dono, H. Tanji, H. Aoki, T. Ide, K. Nishio, C. Gregory, J. Waugh, N. Woolsey, A. Dizire, M. Koenig, H. Ide, K. Tsubouchi, H. Takabe, Interaction of high mach-number shocks in laser-produced plasmas, High Energy Density Physics 9 (1) (2013) 187 - 191.

[11] W. Fox, G. Fiksel, A. Bhattacharjee, P.-Y. Chang, K. Germaschewski, S. X. Hu, P. M. Nilson, Filamentation instability of counterstreaming laser-driven plasmas, Phys. Rev. Lett. 111 (2013) 225002. doi:10.1103/PhysRevLett.111.225002.

[12] S. Davis, R. Capdessus, E. d'Humières, S. Jequier, I. Andriyash, V. Tikhonchuk, Numerical simulations of energy transfer in counter-streaming plasmas, High Energy Density Physics 9 (1) (2013) 231-238.

[13] A. P. Fews, P. A. Norreys, F. N. Beg, A. R. Bell, A. E. Dangor, C. N. Danson, P. Lee, S. J. Rose, Plasma ion emission from high intensity picosecond laser pulse interactions with solid targets, Phys. Rev. Lett. 73 (13) (1994) 1801-1804.

[14] R. A. Snavely, M. H. Key, S. P. Hatchett, T. E. Cowan, M. Roth, T. W. Phillips, M. A. Stoyer, E. A. Henry, T. C. Sangster, M. S. Singh, Intense high-energy proton beams from petawatt-laser irradiation of solids, Phys. Rev. Lett. 85 (14) (2000) 2945-2948.

[15] S. Wilks, A. Langdon, T. Cowan, M. Roth, M. Singh, S. Hatchett, M. Key, D. Pennington, A. MacKinnon, R. Snavely, Energetic proton generation in ultra-intense laser-solid interactions, Physics of Plasmas (1994-present) 8 (2) (2001) 542-549.

[16] J. Fuchs, P. Antici, E. dHumieres, E. Lefebvre, M. Borghesi, E. Brambrink, C. Cecchetti, M. Kaluza, V. Malka, M. Manclossi, et al., Laser-driven proton scaling laws and new paths towards energy increase, Nature physics 2 (1) (2005) 48-54.

[17] L. Robson, P. T. Simpson, R. J. Clarke, K. W. D. Ledingham, F. Lindau, O. Lundh, T. Mccanny, P. Mora, D. Neely, C. G. Wahlström, M. Zepf, P. Mckenna, Scaling of proton acceleration 
driven by petawatt-laser-[ndash] - plasma interactions, Nature Phys. 3 (2007) 58.

[18] A. Mančić, J. Fuchs, P. Antici, S. Gaillard, P. Audebert, Absolute calibration of photostimulable image plate detectors used as (0.5-20mev) high-energy proton detectors, Review of Scientific Instruments 79 (7) (2008) 073301.

[19] J. F. Ziegler, J. P. Biersack, M. Ziegler, SRIM, Lulu.com, 2008.

[20] P. Mora, Plasma expansion into a vacuum, Physical Review Letters 90 (18).

[21] P. Bolton, M. Borghesi, C. Brenner, D. Carroll, C. De Martinis, A. Flacco, V. Floquet, J. Fuchs, P. Gallegos, D. Giove, et al., Instrumentation for diagnostics and control of laser-accelerated proton (ion) beams, Physica Medica 30 (3) (2014) 255-270.

[22] P. Mora, Thin-foil expansion into a vacuum, Physical Review E 72 (5) (2005) 056401

[23] P. Antici, J. Fuchs, M. Borghesi, L. Gremillet, T. Grismayer, Y. Sentoku, E. d'Humières, C. A. Cecchetti, A. Mančić, A. C. Pipahl, T. Toncian, O. Willi, P. Mora, P. Audebert, Hot and cold electron dynamics following high-intensity laser matter interaction, Phys. Rev. Lett. 101 (2008) 105004. doi:10.1103/PhysRevLett.101.105004.

URL http://link.aps .org/doi/10.1103/PhysRevLett.101.105004

[24] P. Korneev, E. d'Humières, V. Tikhonchuk, Collisionless plasma interpenetration in a strong magnetic field for laboratory astrophysics experiments, Physics of Plasmas (1994-present) 21 (2) (2014) 022117.

[25] P. Korneev, E. d'Humières, D. P. Higginson, J. Fuchs, R. Riquier, H. Pepin, J. Beard, B. Pollock, V. Tikhonchuk, Collisionless magnetized plasma interaction in the context of astrophysical experiments, submitted to Plasma Physic and Controlled Fusion.

[26] Y. Sentoku, A. J. Kemp, Numerical methods for particle simulations at extreme densities and temperatures: Weighted particles, relativistic collisions and reduced currents, Journal of Computational Physics 227 (14) (2008) 6846-6861.

[27] T. Vinci, Neutrino (2014)

URL https://github.com/aflux/neutrino 